MATEC Web of Conferences 26, 01011

(2015)

DOI: $10.1051 /$ matecconf/ 20152601011

(C) Owned by the authors, published by EDP Sciences, 2015

\title{
Polarized Raman Spectroscopy and Chemometric Analysis of Micro-crystalline Silicon for Solar Cells
}

\author{
David Adley ${ }^{1, a}$, Tatiana S. Perova ${ }^{1,2, b}$, Eamonn Monaghan ${ }^{3, c}$, and A.R. Ellingboe ${ }^{3, d}$ \\ ${ }^{1}$ Department of Electronic and Electrical Engineering, Trinity College Dublin, Ireland \\ ${ }^{2}$ ITMO University, St.-Petersburg, Russia \\ ${ }^{3}$ School of Physical Sciences, Dublin City University, Ireland
}

\begin{abstract}
Micro-crystalline silicon $(\mu \mathrm{c}-\mathrm{Si}: \mathrm{H})$ is currently under extensive investigation due to it's applications in solar cells. The crystalline content and structural properties of $\mu \mathrm{c}-\mathrm{Si}: \mathrm{H}$ can be determined from several characterisation techniques such as high-resolution transmission electron microscopy (HRTEM), X-Ray diffraction (XRD), spectroscopic ellipsometry (SE) and Raman spectroscopy. In this paper a solution is proposed to reduce the effect of second order phonons on the Raman spectra of $\mu \mathrm{c}-\mathrm{Si}: \mathrm{H}$ for evaluation of crystalline volume fraction $\chi_{\mathrm{c}}$.
\end{abstract}

\section{Introduction}

Hydrogenated microcrystalline Silicon $(\mu \mathrm{c}-\mathrm{Si}: \mathrm{H})$, consists of small crystalline grains embedded in an amorphous matrix. It has a higher doping efficiency requiring less Hydrogen than amorphous hydrogenated $\mathrm{Si}(\mathrm{a}-\mathrm{Si}: \mathrm{H})$ so is resistant to the Staebler-Wronski effect and has increased absorption efficiency at longer wavelengths than a-Si:H [1]. It has strong potential in multijunction solar cells. Multijuction cells consist of layers of a-Si:H and $\mu \mathrm{c}-\mathrm{Si}: \mathrm{H}$ sandwiched together using their different absorption characteristics to capture as much of the incident light spectrum as possible. VHF-PECVD has been shown to produce high quality crystalline material at high deposition rate [2]. Since the optical and electrical properties of $\mu \mathrm{c}-\mathrm{Si}: \mathrm{H}$ depend on the fraction of crystalline material, the crystallite size, and the grain boundaries an accurate determination of the crystalline volume fraction for $\mu \mathrm{c}-\mathrm{Si}: \mathrm{H}$ is of primary importance. Polarized micro-Raman spectroscopy and Chemometric analysis of Raman spectra of numerous samples of $\mu \mathrm{c}-\mathrm{Si}: \mathrm{H}$ were employed in this paper in order to determine the more reliable technique for sample characterisation.

\section{Experimental}

\subsection{Sample fabrication}

A large selection of $\mu \mathrm{c}-\mathrm{Si}: \mathrm{H}$ samples deposited on glass substrates by VHE-PECVD (30-300 MHz) having varying crystalline volume fractions were used as a sample set. This set of samples covering the complete crystallinity spectrum were manufactured by adjusting the silane and hydrogen ratio during $\mu \mathrm{c}-\mathrm{Si}: \mathrm{H}$ film deposition (see Ref.[2] for details).

\subsection{Characterisation}

Raman spectra were registered in a backscattering geometry from the samples using a RENISHAW 1000 micro-Raman system under $514 \mathrm{~nm}$ (Ar+ laser) and 633 $\mathrm{nm}$ (He-Ne laser) excitations. The use of different excitation wavelengths allows the Raman signal to be collected at different depths of penetration.

\section{Results and discussion}

The examples of Raman spectra registered from $\mu c-S i: H$ samples with different $\chi_{c}$ values are shown in Fig. 1 (a). It can be seen that these Raman spectra composed of a sharp peak centred at $\sim 520 \mathrm{~cm}^{-1}$, assigned to the microcrystalline part of the film, and of a broad band around $\sim 485 \mathrm{~cm}^{-1}$, attributed to the amorphous Si phase. The crystalline volume fraction, $\chi_{c}$ is normally determined as the ratio of the integrated intensities of LO Raman band of crystalline Si at $\sim 520$ $\mathrm{cm}^{-1}$ and the TO band of amorphous $\mathrm{Si}(\mathrm{a}-\mathrm{Si})$ at 485 $\mathrm{cm}^{-1}$. As these bands overlap several techniques have been developed to deconvolute the spectra into its separate components, using typically two and three peaks fitting. Two peaks fitting assigns one Gaussian peak to the amorphous component and a second Lorentzian peak to the crystalline component of the $\mu \mathrm{c}-\mathrm{Si}: \mathrm{H}$ spectrum. Furthermore, the amorphous

\footnotetext{
adleyd@tcd.ie, ${ }^{\mathrm{b}}$ perovat@tcd.ie, ${ }^{\mathrm{c}}$ bjorn.mt@gmail.com, ${ }^{\mathrm{d}}$ bert.ellingboe@dcu.ie
} 
component is not represented by a single Gaussian function but rather a combination of optical and acoustic phonon bands [3], therefore the Gaussian function does not adequately fit the spectra without significant error. Adding a further peak, intermediate between the amorphous peak and the crystalline peak as shown in Fig. 1(b), significantly reduces the residual error, however there is little evidence of a physical explanation to account for this additional peak [4].

If three peaks fitting is used the $\chi_{c}$ is then determined from Eq. (1)

$$
\chi_{c}=\left(I_{c}+I_{m}\right) /\left(I_{a}+I_{m}+\chi_{c}\right),
$$

where $I_{a}, I_{m}$ and $I_{c}$ are the amorphous, intermediate and crystalline Raman integrated intensities, and $\gamma$ is a wavelength dependent scaling factor taken to be on average 0.88 [3]. It is extremely difficult to reliably and repeatably fit the amorphous and intermediate peaks. Smit et al. [3] proposed an alternative technique in which the shape of the amorphous component was considered constant across samples of varying crystallinity and the change in the spectra was primarily due to changes in the crystalline peak. All peaks surrounding the first order scattering between approximately 200 and $440 \mathrm{~cm}^{-1}$ are assigned to the amorphous spectrum. A 'least squares fit' was then applied in order to scale a reference amorphous spectra so that it could then be subtracted from the sample spectra.

The remaining spectra could be assigned to the microcrystalline component. The crystalline volume fraction was then found as

$$
\chi_{c}=I_{c} /\left(I_{a}+\chi_{c}\right),
$$

where $I_{c}$ is the integrated intensity of the spectrum remaining after the amorphous component subtraction, this corresponds to the microcrystalline component. $I_{a}$ is the TO component of the amorphous spectrum, the TO component can be found approximately by fitting with three Gaussian curves (see [3] for details).

This was significantly more reliable than the two and three peaks fitting approaches. However this technique suffers from several problems. The crystalline component does not consist solely of the first order Raman peak at approximately $520 \mathrm{~cm}^{-1}$ but also includes second order peaks of Raman scattering including overtone, combination and acoustic phonon peaks at wavenumbers above and below the central TO peak at $\sim 520 \mathrm{~cm}^{-1}$ [5]. The influence of these second order peaks is more pronounced for longer wavelengths of excitation such as $633 \mathrm{~nm}$ due to its larger penetration depth. As these peaks result from the crystalline phase, they are most pronounced when the sample has a large crystalline component. The second order peaks overlap the regions assigned purely to the amorphous spectra and used to calculate the scaling factor in [3]. Therefore the correct scaling factor cannot be found using the least fit analysis described in [3] due to this overlap and leading to errors as shown in Fig. 2 (a).
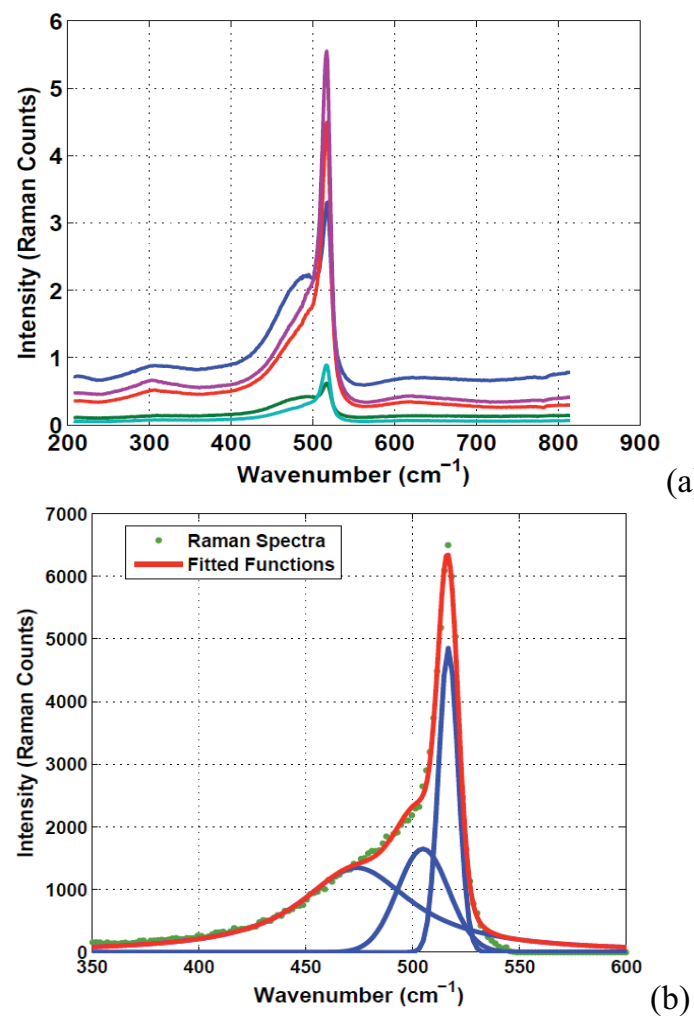

Figure 1. a) Raman spectra of investigated $\mu \mathrm{c}-\mathrm{Si}: \mathrm{H}$ sample with different crystalline volume fraction, b) example of fitting of Raman spectrum with 2 Gaussian and 1 Lorentzian functions (three peaks fitting).

In order to find the correct multiplier or scaling factor for spectra subtraction, the crystalline second order phonon peaks must be suppressed or removed. In single crystal $\mathrm{Si}$ the contribution of the acoustical phonons can be substantially suppressed by using polarized Raman measurements in $\mathrm{VH}$ or [001(100,010)00-1] geometry (see [6] for details). However, in $\mu \mathrm{c}-\mathrm{Si}: \mathrm{H}$ with a very high crystalline volume fraction, a much reduced second order spectrum was still visible despite attenuating polarisation filters, particularly in the region at $300 \mathrm{~cm}^{-1}$. At the same time the amorphous peak at $630 \mathrm{~cm}^{-1}$ does not have this problem. Therefore, this peak was used to obtain the scaling factor. The a-Si spectrum is then scaled by this factor and subtracted from the microcrystalline $\mathrm{Si}$ spectrum as shown in Fig. 2 (b). Table 1 shows the results of the crystalline volume fraction $\chi_{\mathrm{c}}$ calculations using different fitting techniques discussed above. It can be seen from the Table 1 that the two peaks fitting results in significantly smaller $\chi_{\mathrm{c}}$ values than obtained from the other techniques in particular for the larger $\chi_{\mathrm{c}}$. The fitting using the $630 \mathrm{~cm}^{-1}$ peak shows a higher $\chi_{c}$ value in general than the other columns as it is less effected by the contribution from the second order Raman peaks which tend to promote low crystallinity values. This technique can also be applied using 
non-polarised light to improve the resolution of the Smit's [3] approach.
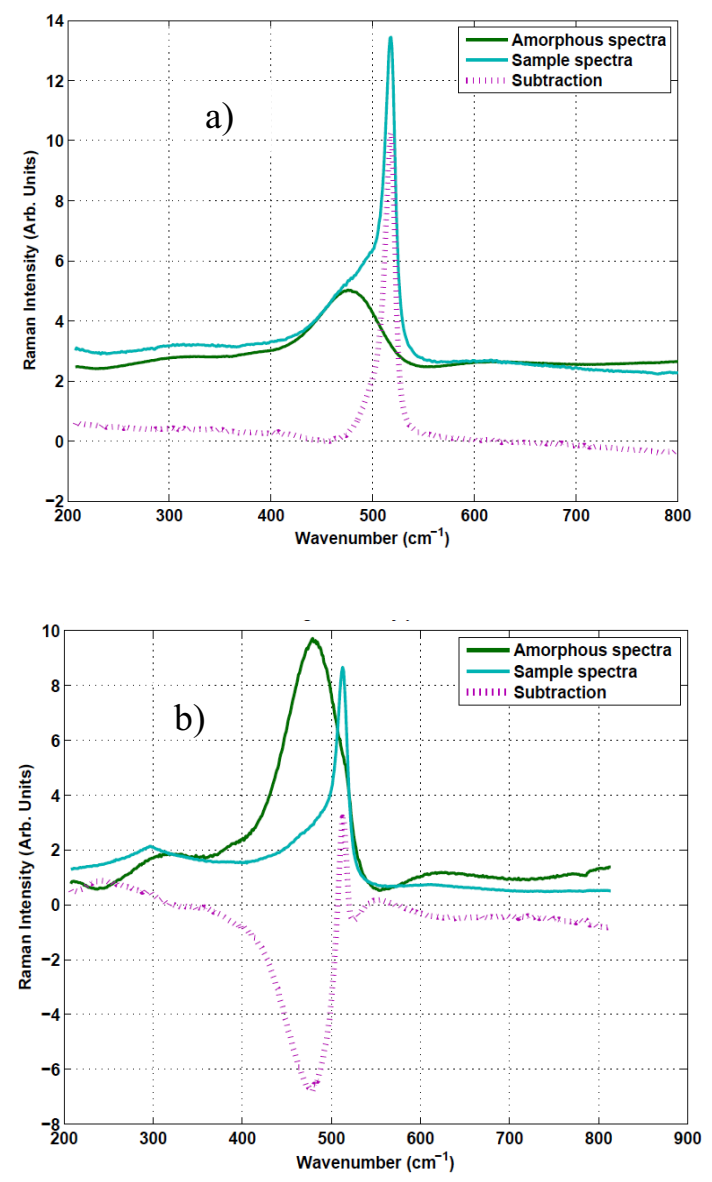

Table 1. Calculations of $\chi_{\mathrm{c}}$ values using Eqs. (1) and (2) and Smit's [3] approach.
Figure 2. Subtraction of Raman spectra of amorphous Si and $\mu \mathrm{c}-\mathrm{Si}: \mathrm{H}:$ a) non-polarized and b) polarized Raman spectra.

An alternative technique is to use regression analysis in particular Classical Least Squares (CLS) to determine the scaling factor. The CLS model assumes that measurements are the weighted sum of linearly independent signals. In spectroscopy, the CLS model assumes that measured spectra are the sum of pure component spectra weighted by their respective concentration. Thus, the model is $x=p S$, where $x$ is the measured spectra, $S$ is the matrix of pure component or basis spectra and $\boldsymbol{p}$ is the vector containing the concentrations of the component spectra or loadings. Generally, given the vector of measurements, $x$, one would like to know $\boldsymbol{p}$, the degree to which each component contributes to the overall measurement. Of the chemometric techniques tested this was found to be among the simplest and best. The Eigenvector PLS Toolbox [7] was used for CLS fitting. Initially the dataset was pre-processed using SavGol smoothing, second order polynomial baseline fitting, and area normalisation. The scaling factor for each individual $\mu \mathrm{c}-\mathrm{Si}: \mathrm{H}$ spectrum is found in the loading matrix $(\boldsymbol{p})$ consisting of two factors corresponding to the amorphous and crystalline components. The amorphous factor can then be used as the scaling factor in the technique described in [3]. Table 2 displays the results of this approach.

\begin{tabular}{|l|l|l|l|l|l|l|l|l|}
\hline \multirow{2}{*}{$\begin{array}{l}\text { Sample } \\
\text { Name }\end{array}$} & \multicolumn{2}{|c|}{ Fitting with } & \multicolumn{2}{l|}{ Smit's approach } & $\begin{array}{l}\text { Sample } \\
\text { Name }\end{array}$ & CLS & $\begin{array}{l}\text { Smit's } \\
\text { model, } \\
630 \mathrm{~cm}^{-1}\end{array}$ & $\begin{array}{l}\text { Two } \\
\text { peaks } \\
\text { fit }\end{array}$ \\
\cline { 2 - 9 } & 2 peaks & 3peaks & $\begin{array}{l}630 \mathrm{~cm}^{-1} \\
\text { peak }\end{array}$ & both peaks & & & & \\
\hline S1 & 0.41 & 0.57 & 0.71 & 0.70 & S11 & 0.36 & 0.48 & 0.37 \\
S2 & 0.42 & 0.59 & 0.68 & 0.67 & S12 & 0.25 & 0.35 & 0.26 \\
S3 & 0.20 & 0.40 & 0.28 & 0.32 & S13 & 0.20 & 0.28 & 0.24 \\
S4 & 0.35 & 0.52 & 0.63 & 0.60 & S14 & 0.15 & 0.24 & 0.18 \\
S5 & 0.27 & 0.45 & 0.53 & 0.48 & S15 & 0.12 & 0.18 & 0.13 \\
S6 & 0.44 & 0.59 & 0.72 & 0.68 & S16 & 0.09 & 0.14 & 0.10 \\
S7 & 0.47 & 0.60 & 0.46 & 0.46 & S17 & 0.04 & 0.12 & 0.09 \\
S8 & 0.21 & 0.43 & 0.45 & 0.44 & S18 & 0.02 & 0.08 & 0.07 \\
\hline
\end{tabular}

$\mathrm{cm}^{-1}$ is used to determine the scaling factor then the CLS method should provide the best results.

\section{Summary}

Both the polarisation and chemometric techniques significantly attenuates the influence of the second order phonons and enables for more accurate calculation of the crystalline volume fraction for highly crystalline samples. The chemometric technique requires significantly shorter exposure time and could potentially be used to rapidly map large regions of the sample under investigation. If the sample is highly crystalline and only the phonon at 630

\section{References}

1. G. Parascandolo, G. Bugnon, A. Feltrin and C. Ballif, High-rate deposition of microcrystalline silicon in a large-area PECVD reactor and integration in tandem solar cells, Prog. Photovolt. Res. Appl. 18 (2010) 257-264.

2. E. Monaghan, T. Michna, C. Gaman, D. O'Farrel, K. Ryan, D. Adley, T.S. Perova, B. Drews, M. Jaskot, A.R. Ellingboe, Characterisation of thin silicon films 
deposited by plasma enhanced chemical vapour deposition at $162 \mathrm{MHz}$, using a large area, scalable, multi-tile-electrode plasma source, Thin Solid Films, 519 (2011) 6884-6886.

3. C. Smit, R.A.C.M.M. Van Swaaij, H. Donker, A.M.H.N. Petit, W.M.M. Kessels, and M.C.M. Van de Sanden, Determining the material structure of microcrystalline Silicon from Raman spectra, J. Appl. Phys., 94 (2003) 3582-3588.

4. M. Ledinsky, A. Vetushka, J. Stuchlik, T. Mates, A. Fejfar, J. Kocka, and J. Stepnek, Crystallinity of the mixed phase silicon thin films by Raman spectroscopy, J. Non-Cryst. Sol., 354 (2008) 2253-2257.

5. P. Temple and C. Hathaway, Multiphonon Raman spectrum of silicon, Phys. Rev. B, 7 (1973) 3685-3697.

6. A. V. Baranov, A. V. Fedorov, T. S. Perova, S. Solosin, R. A. Moore, V. Yam, D. Bouchier and V. Le Thanh, Polarized Raman spectroscopy of multilayer $\mathrm{Ge} / \mathrm{Si}$ (001) quantum dot heterostructures, J.Appl.Phys., 96 (2004) 2857-2863.

7. Information on http://www.eigenvector.com. 